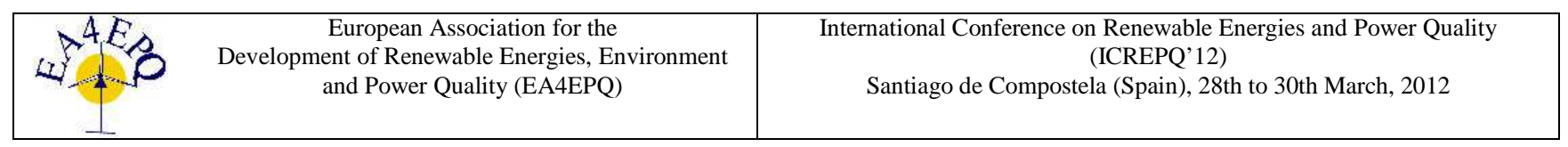

\title{
Wind Farm and System Modelling Evaluation in Harmonic Propagation Studies
}

\author{
G. A. Mendonça ${ }^{1}$, H. A. Pereira ${ }^{1,2}$ and S. R. Silva ${ }^{1}$ \\ ${ }^{1}$ Graduate Program in Electrical Engineering - Universidade Federal de Minas Gerais \\ Av. Antônio Carlos 6627, 31270-901, Belo Horizonte, MG, Brazil \\ Phone: +55-31-3409-4842, e-mail: gforti@gmail.com, heverton.pereira@ufv.com.br, selenios@ dee.ufmg.br \\ ${ }^{2}$ Department of Electrical Engineering, Universidade Federal de Viçosa \\ Av. P.H.Rolfs S/N,36570-000, Viçosa, MG, Brazil
}

\begin{abstract}
Wind power plants are playing an important role in renewable energy generation in this decade. With the enhancements in its technology, mainly based on the aggregation of power electronics, many studies have been carried for evaluating their impact in power quality. Although the Brazilian Electrical System National Operator, who is responsible for transmission and generation system management, has suggested a procedure for such studies, there are many aspects related to the simulation algorithm and the electrical components modelling that are left aside from the problem, without an adequate reasoning of its impacts on power quality simulation results.
\end{abstract}

This paper presents a detailed analysis of a wind farm impact on the Brazilian distribution system power quality. Both internal (wind park elements) and external (power grid) components modelling effects on harmonic propagation are considered, and these effects are evaluated

\section{Key words}

Wind Farm, Harmonic Analysis, Frequency Domain Simulation, Power System Modelling, Simulation Software;

\section{Introduction}

Adequate modelling of electrical components has always been a concern when analysing harmonic penetration. Several works had been carried out in order to investigate the problems incurred from evaluating harmonic studies with a comprehensive analysis of the used approach.

Many of these studies, [1]-[4], discuss the methods which can be used to evaluate harmonic distortion: single and three phase system representation, time and frequency domain, etc. These works have lead to what is considered to be the most important aspect in harmonic studies, a sense of how these methods can affect the quality of the results. But it does not state the difference in improvement from one to another in a quantity matter.

Another important subject when dealing with harmonic propagation is the electrical components modelling. The difficulty in finding the proper equivalent to represent the system's main equipment without letting the problem become neither too complex, impracticable to simulate, nor too simple, with inaccurate results.

Using a wind power plant as the study case, a sensibility analysis can evaluate the impact of the assumptions that one can make in this sort of study. For that, two computer simulation programs will be used. The Alternate Transient Program - ATP, which has several available equipments models for applications ranging from steady state to very complex transient analysis, will be used for comparing component equivalents with different complexity degree. On the other hand, DIgSILENT PowerFactory, an engineering tool specifically designed for Power System Analysis, will be used to compare the effects of systems degrees of representation.

\section{System Description}

The studied wind is similar to one of the 54 wind parks connected to the Brazilian grid. It consists of a collector substation and three feeders with a total of 28 wind generators. The wind farm system, which operates with a voltage of $34.5 \mathrm{kV}$, is connected to the grid through a step-up transformer, $34.5 / 69 \mathrm{kV}$, and a transmission line with $21 \mathrm{~km}$. Then, a substation elevates the voltage to $230 \mathrm{kV}$ in order to connect wind farm with the primary transmission system.

In that wind farm, the wind turbine generators - WTG are connected in three parallel groups, one composed of ten and two of nine units. Figure 1 illustrates the first group with ten wind generators. The other two groups are constructed analogously. Each wind generator consists of a permanent magnet synchronous generator - PMSG, with a full converter, each unit been capable of generating up to $1.5 \mathrm{MVA}$. In order to meet power quality requirements, the converter is followed by a second-order low-pass filter that helps smoothing the voltage waveform. This LC filter is composed of a series $0.15 \mathrm{mH}$ inductor and a shunt $500 \mu \mathrm{F}$ capacitor per phase. A scheme illustrating the PMSG is pictured in Figure 2. 


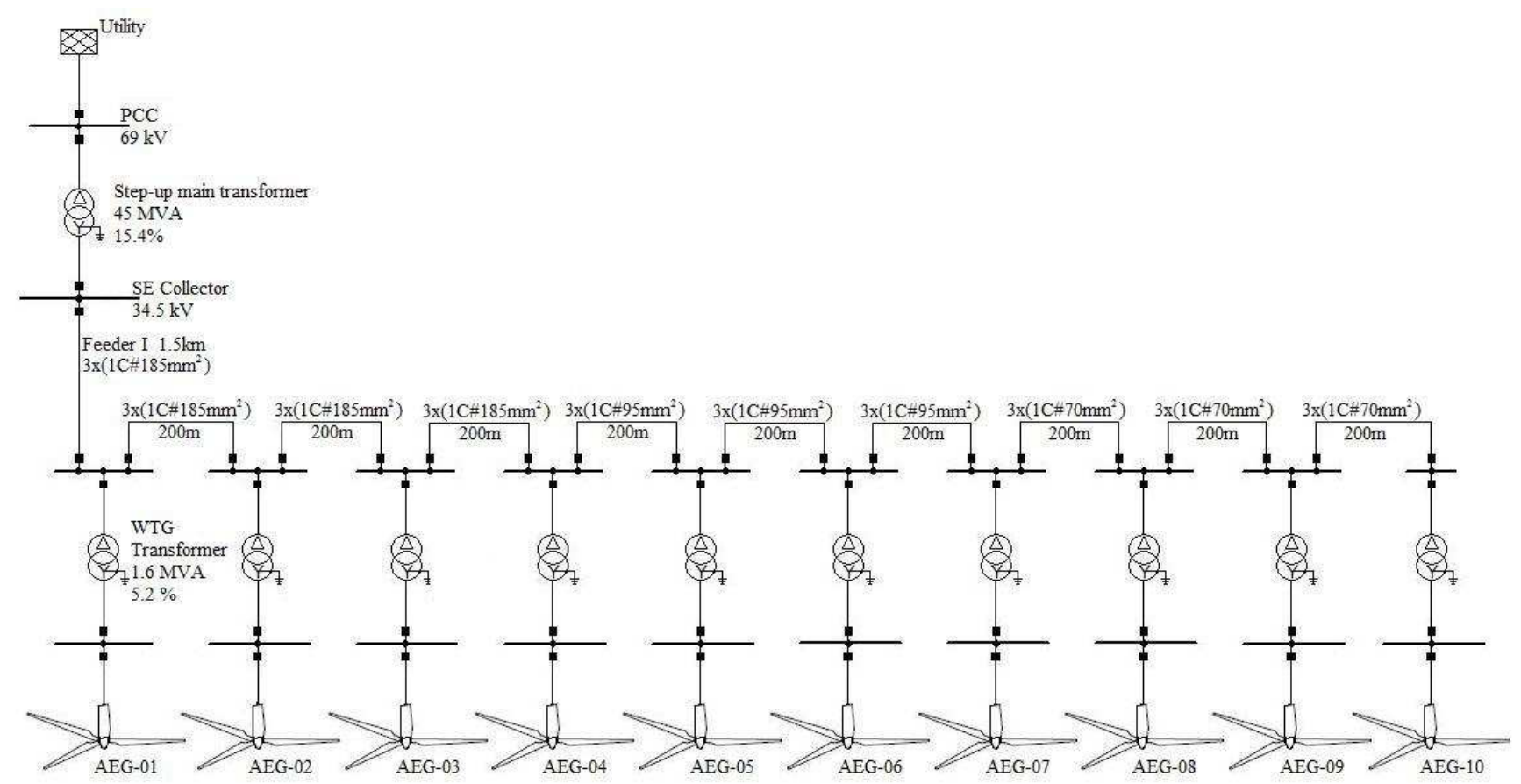

Fig. 1. One-line diagram for Group I WTG of the simulated wind park

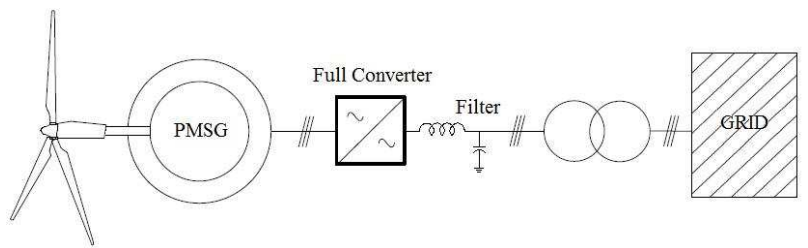

Fig. 2. Wind turbine with PMSG, back-to-back PWM converter and output filter

Although the wind generator technology affects substantially the harmonic analysis, this paper will consider one technology, modelled as harmonic current source. It will help concentrate in how the system modelling affects the overall result, considering only one technology and its harmonic current spectrum, listed in Table I.

The electrical components parameters of the simulated wind park were obtained from commercial wind farms, manufacturer engineering catalogue and electrical standards. Electrical cable and transformer's characteristics are presented in Tables II and III, respectively.

\section{Electrical Equipment Modelling}

For the modelling of the electrical system, the following assumptions are considered:

1) All electrical supplies are balanced

2) The system components are symmetrical

3) All wind turbines are equal

Having considered that, the wind turbines were modelled as harmonic current sources without specifying the harmonic phase angle spectrum. As recommended by [5], the lack of diversity presented in most wind parks could be interpreted as a high probability of the harmonics to be in phase. Also, since all electrical components are symmetrical, the system will be treated as single-phase one.

Table I. - WTG Harmonic Spectrum

\begin{tabular}{|c|c|c|}
\hline Harmonic Order & Current [A] & $\mathrm{I}_{\mathrm{h}} / \mathrm{I}_{1}[\%]$ \\
\hline 1 & 1396.815 & $100 \%$ \\
\hline 2 & 4.78985 & $0.343 \%$ \\
\hline 4 & 2.80642 & $0.201 \%$ \\
\hline 5 & 6.08122 & $0.435 \%$ \\
\hline 7 & 6.57453 & $0.471 \%$ \\
\hline 8 & 5.60740 & $0.401 \%$ \\
\hline 10 & 7.61141 & $0.545 \%$ \\
\hline 11 & 20.33810 & $1.456 \%$ \\
\hline 13 & 25.89040 & $1.854 \%$ \\
\hline 14 & 6.62944 & $0.475 \%$ \\
\hline 16 & 5.17552 & $0.371 \%$ \\
\hline 17 & 10.62870 & $0.761 \%$ \\
\hline 19 & 5.87022 & $0.420 \%$ \\
\hline 20 & 4.53000 & $0.324 \%$ \\
\hline 22 & 4.60657 & $0.330 \%$ \\
\hline 23 & 5.15894 & $0.369 \%$ \\
\hline 25 & 3.28080 & $0.235 \%$ \\
\hline 26 & 2.79949 & $0.200 \%$ \\
\hline 28 & 1.85472 & $0.133 \%$ \\
\hline 29 & 3.78464 & $0.271 \%$ \\
\hline 31 & 3.35227 & $0.240 \%$ \\
\hline 35 & 4.88246 & $0.350 \%$ \\
\hline 37 & 3.61668 & $0.259 \%$ \\
\hline 41 & 2.19004 & $0.157 \%$ \\
\hline 43 & 1.50464 & $0.108 \%$ \\
\hline 50 & 1.47892 & $0.106 \%$ \\
\hline
\end{tabular}

Table II. - Conductor Electrical Parameters

\begin{tabular}{|c|c|c|c|}
\hline \multicolumn{4}{|c|}{ XLPE 20/35 kV Cables } \\
\hline Size $\left[\mathrm{mm}^{2}\right]$ & $\mathrm{R}_{\mathrm{CA}}[\Omega / \mathrm{km}]$ & $\mathrm{X}_{\mathrm{L}}[\Omega / \mathrm{km}]$ & $\mathrm{X}_{\mathrm{C}}[\Omega / \mathrm{km}]$ \\
\hline 185 & 0.130 & 0.145 & $13,527.0$ \\
\hline 95 & 0.248 & 0.160 & $16,713.0$ \\
\hline 70 & 0.344 & 0.168 & $18,433.0$ \\
\hline
\end{tabular}


Table III. - Transformer Specification and Parameters

\begin{tabular}{|c|c|}
\hline \multicolumn{2}{|c|}{ Step-up main transformer } \\
\hline Rated Power & $45 \mathrm{MVA}$ \\
\hline Voltage & $69 / 34.5 \mathrm{kV}$ \\
\hline Connection & Dyn 1 \\
\hline Z & $15.4 \%$ \\
\hline LTC & $\pm 8 \times 1.25 \%$ \\
\hline \multicolumn{2}{|c|}{ Generator transformer } \\
\hline Rated Power & $1.6 \mathrm{MVA}$ \\
\hline Voltage & $34.5 / 0.62 \mathrm{kV}$ \\
\hline Connection & Dyn 1 \\
\hline Z & $5.2 \%$ \\
\hline LTC & $\pm 2 \times 2.25 \%$ \\
\hline
\end{tabular}

Focusing in the degree of system representation and the equipment modelling, the next sections will discuss them in detail.

\section{A. Cable Modelling}

The distribution system of the wind farm is composed by single-core XLPE cables with the conductor size ranging from $70 \mathrm{~mm}^{2}$ to $185 \mathrm{~mm}^{2}$. The parameters were obtained from manufacture catalogues and used to feed the models.

The electrical parameters listed in these catalogues, which are usually gathered from measured data, were evaluated against a simulated model. In [6], the author discusses the modelling of these cables in EMTP-type programs, e.g. ATP, delineating a procedure which helps to overcome all inaccuracies that the model might present. This procedure was concerned mainly with transient analysis. Thus, it concentrates on providing common materials properties, on representing semiconductor screens properly, on analysing the significance of grounding condition of sheath, etc. Therefore, the electrical parameters calculated according with this procedure can be used to assess the accuracy of catalogue data.

A XLPE single-core $300 \mathrm{~mm}^{2}$ cable was modelled according with [6], at ATP's Line/Cable Constant - LCC routine, and the distributed line component. The maximum error found for both amplitude and phase angle was $4.02 \%$ and $1.47 \%$, respectively.

Therefore, the LCC model present in ATP gives a better response for transient phenomena, but for limited frequency range simulations, such as harmonic analysis, the simpler distributed parameter model is sufficient.

\section{B. Transformer Modelling}

Usually, in steady-state studies, e.g. short-circuit and load flow, transformers are modelled simply by a series impedance. Considerations relating winding stray capacitance are generally made for higher frequency studies, where some authors state that its effects are only noticeable for frequencies higher than $4 \mathrm{kHz}$ [1].

This assumption can be validated using ATP. The program has several transformer models available, but the most complete one is the hybrid transformer, which represents stray capacitance. Typical values for this parameter can be found in [7].

For the analysis up to $3 \mathrm{kHz}$, a simple voltage divider circuit simulation showed that the maximum difference between the results with and without the capacitance effect was $1.67 \%$ for the amplitude and $3.86 \%$ for the phase angle.

\section{Distribution and Transmission System Modelling}

What degree of representation should be considered as accurately sufficient? This question always bothered when investigating broader frequency spectrum problems. If representing the entire network is impractical, estimating its behaviour from point of common coupling - PCC, short-circuit impedance, as used sometimes, is unrealistic [4].

In [4], the author suggests a system equivalent which is based on the prominence of low order resonances. It represents the system impedance with an L-C-L equivalent circuit, estimated from short-circuit impedance and the first two resonant frequencies, i.e. parallel and series.

The Electric System National Operator (ONS in Portuguese) is the entity responsible for coordinating and controlling the operation of generation and transmission facilities in the National Interconnected Power System. It offers information on Brazilian's system, including electrical parameters of transmission line, transformer, capacitor bank, etc. It also provides the system data base built in the programs developed by CEPEL. The data base can be converted for harmonic analysis program, HarmZs, to find the frequency response at any bus compounding the Brazilian grid.

For this study, a few considerations were made in order to simplify the analysis. All transmission lines are modelled as a single equivalent nominal $\pi$-model, all machines impedances were neglected and all loads were modelled as parallel loads. The frequency response observed in the PCC is pictured in Figure 3, which also shows the frequency response obtained from the shortcircuit parameters and with the L-C-L equivalent circuit calculated according to [4]

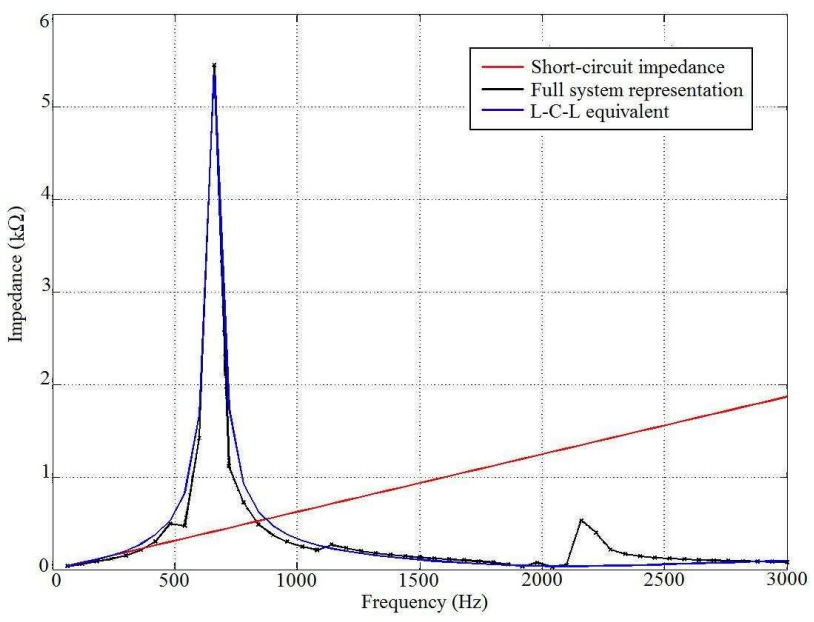

Fig. 3. System frequency response at the PCC 
With the system data collected from the ONS database, the primary transmission was modelled in PowerFactory with eight, fifteen and nineteen buses. In each case, the frequency response was obtained with two types of representations of the part of system not explicitly modelled: short-circuit impedance and L-C-L circuit equivalent at each boundary bus. Table IV illustrates the maximum absolute error observed for the resonance amplitude and frequency in each approach when compared with the entire system response.

Table IV. - System representation error

\begin{tabular}{|c|c|c|c|c|}
\hline \multirow{2}{*}{$\begin{array}{c}\text { Number of } \\
\text { Buses }\end{array}$} & \multicolumn{4}{|c|}{ Maximum Impedance Error } \\
\cline { 2 - 5 } & Short-circuit impedance & \multicolumn{2}{|c|}{ L-C-L equivalent } \\
\hline 1 & $972.0 \%$ & $1260 \mathrm{~Hz}$ & $61.9 \%$ & $660 \mathrm{~Hz}$ \\
\hline 8 & $60 \%$ & $600 \mathrm{~Hz}$ & $37.8 \%$ & $660 \mathrm{~Hz}$ \\
\hline 15 & $62.5 \%$ & $600 \mathrm{~Hz}$ & $32.6 \%$ & $660 \mathrm{~Hz}$ \\
\hline 19 & $10.4 \%$ & $660 \mathrm{~Hz}$ & $6.4 \%$ & $660 \mathrm{~Hz}$ \\
\hline
\end{tabular}

The prior analysis also considered secondary distribution system, representing lower voltage equipment. Thus, for a sensibility assessment, a second simulation was carried out. Starting with the representation which incurred the best result, with nineteen EHV buses, the system was simulated without any equipment rated lower than $230 \mathrm{kV}$. Then, the system complexity was slightly increased by representing the components connected to the point of common coupling. The comparison of these two results is summarized in Table V.

Table V. - System representation error

\begin{tabular}{|c|c|c|c|}
\hline \multicolumn{4}{|c|}{ Maximum Impedance Error } \\
\hline \multicolumn{3}{|c|}{ EHV System Response } & \multicolumn{2}{|c|}{ PCC System Modelled } \\
\hline $39.8 \%$ & $660 \mathrm{~Hz}$ & $8.3 \%$ & $660 \mathrm{~Hz}$ \\
\hline
\end{tabular}

As the high voltage transmission system has lower losses, its impedance dominates the frequency response. But, it's not necessary to model accurately the entire primary transmission network. The reduced system frequency response converged to the expected result with nineteen buses. As for the secondary distribution system, the model should represent at least those equipments which are connected to the PCC.

\section{Full Converter PMSG Turbine Modelling}

For the present harmonic propagation study, the wind turbines are modelled as harmonic current source in parallel with a fundamental frequency source [8]. The synchronous machine parameters were set in order to give the fundamental power, $1.5 \mathrm{MW}$, without affecting the impedance frequency spectrum. Figure 6 illustrates the model used in PowerFactory.

The harmonic current listed on Table I was collected through measurements made according to IEC 61400-21. Since it is concerned the WTG power quality impact, all quantities were collected after the converter filter, before the transformer's low voltage side. Thus, the harmonic spectrum obtained from turbine manufactures already considers the filter effect, but not its influence over the system's impedance. Neglecting this effect could under or overestimate some dangerous resonant frequencies.

Therefore, the harmonic current spectre will be recalculated according to equation (1). The harmonic current injection, modelled as illustrated in Figure 4, will be modified according to Figure $5 . \mathrm{Z}_{\text {grid }}$ is the short circuit impedance at the $34.5 \mathrm{kV}$ bus.

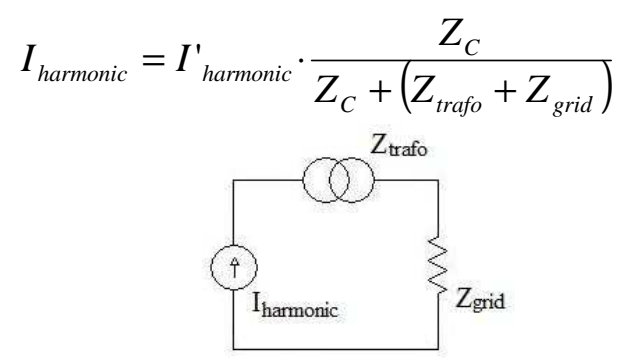

Fig. 4. WTG model

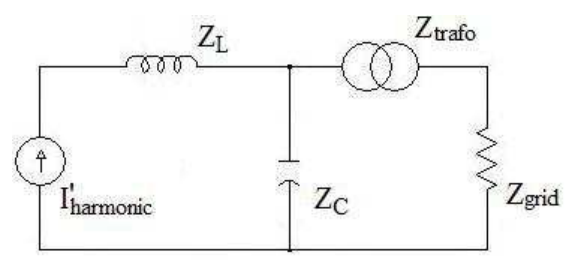

Fig. 5. WTG model

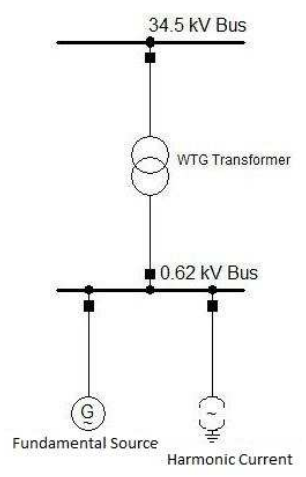

Fig. 6. WTG model

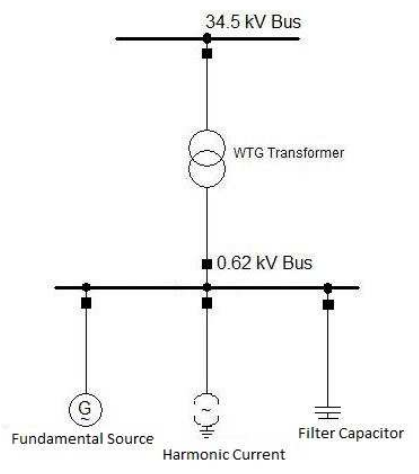

Fig. 7. Modified WTG model
The filter gain is illustrated in Figure 8. For this study, two values of short-circuit power were used: 150 MVA and 1500 MVA. The first value used is the short-circuit impedance at the $34.5 \mathrm{kV}$ bus for the study case system. The second value was used to find the impact of this parameter in the overall result, estimating the situation during IEC testing to obtain the current spectrum.

From (1), it can be seen that the filter inductor has no effect on the output current. The modified WTG model that will be simulated in PowerFactory is illustrated Figure 7.

\section{E. Aggregate Model for Large Wind Power Plants}

Finding an aggregated model for wind power plant to simplify the analysis has become a powerful tool when investigating large wind farms. IEC [5] proposes an aggregation method based on the assumption that the wind turbines and their converters are unequal. However, most wind farms turbines have equal technologies. 


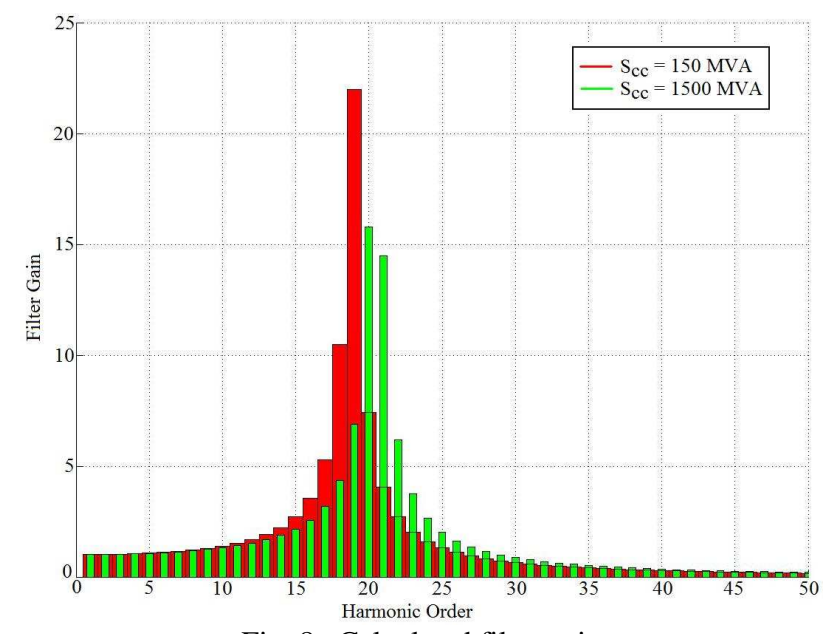

Fig. 8. Calculated filter gain

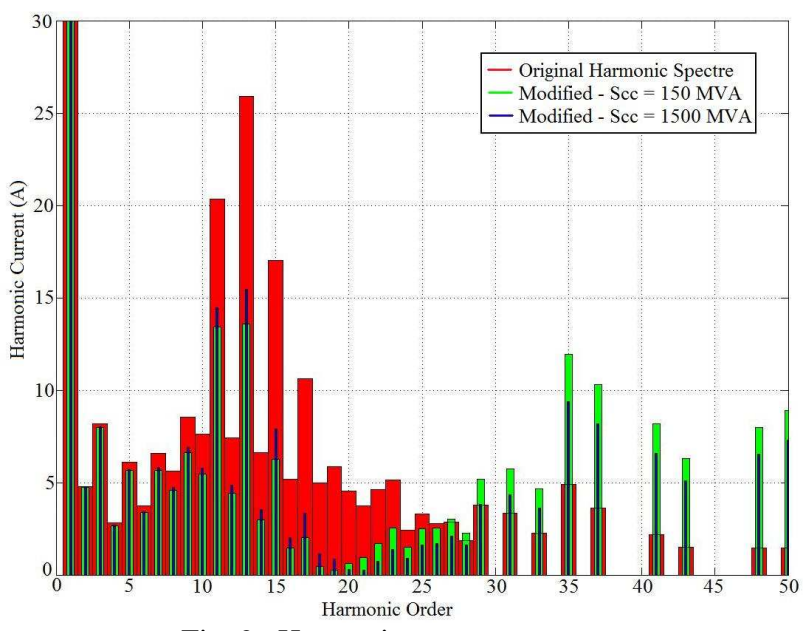

Fig. 9. Harmonic current spectrum

The limits that this method implies are very well discussed in [9], where the author proposes a method of aggregation using two-port network theorem, the equivalent $\mathrm{ABCD}$ parameters are obtained from individual cable parameter, which also modify the harmonic current injection. The wind park is composed with $n$ cable sections, as illustrated Figure 10, and does not considers the generator transformer. The wind turbine current emissions are reflected to the high-voltage side of the transformer.

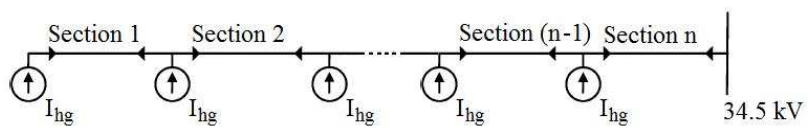

Fig. 10. Wind turbine group for aggregation method

A second circuit simplification is discussed in [10], where the derivation is based on apparent power losses. In this approach the author scales the transformer impedance by the number of units. The results obtained from both methods are discussed in the next section, where the wind farm system is studied.

\section{Study Case}

The Wind Power System studied in the present paper is partially illustrated in Figure 1. First, it was simulated so that the two aggregation methods discussed previously could be evaluated.
As stated in [9], the first method is based on two-port network theory where the medium-voltage network is modelled by its linear passive elements. The harmonic current injection is considered on the high side of the WTG. Therefore, generator transformers are not considered. For the second method, all transformers were scaled according with the number of parallel units.

The impedance frequency response seen from the 34.5 $\mathrm{kV}$ bus was compared for the three cases: the system with 10 WTG and the aggregated equivalent using both methods. The maximum error found in each case was $8.32 \%$ and $21.74 \%$, respectively. Secondly, the total harmonic distortions - THD, observed in the $34.5 \mathrm{kV}$ and the $69 \mathrm{kV}$ buses are listed in Table VI.

Considering both methods with and without the filter explicitly modelled, the results were compared through the $34.5 \mathrm{kV}$ and the $69 \mathrm{kV}$ bus THD values. Table VI gives the results.

The harmonic current injection was modified and the filter's capacitor was explicitly modelling in each case, except for the first method of aggregation, which only uses the current seen from the high-voltage side of the transformers. The THD results for this simulation are also presented in Table VI.

Table VI. - Total Harmonic Distortion

\begin{tabular}{|c|c|c|}
\hline \multirow{3}{*}{ 10 Turbines Explicitly Modelled } \\
\hline \multirow{2}{*}{ Bus } & \multicolumn{2}{|c|}{ THD [\%] } \\
\cline { 2 - 3 } & Filter Effect Neglected & Filter Modelled \\
\hline $34.5 \mathrm{kV}$ & 16.60 & 5.63 \\
\hline $69 \mathrm{kV}$ & 9.08 & 3.42 \\
\hline & \multicolumn{2}{|c|}{ Thgregation - Method I } \\
\hline \multirow{2}{*}{ Bus } & \multicolumn{2}{|c|}{ THD } \\
\cline { 2 - 3 } & Filter Effect Neglected & Filter Modelled \\
\hline $34.5 \mathrm{kV}$ & 17.92 & - \\
\hline $69 \mathrm{kV}$ & \multicolumn{2}{|c|}{ THD [\%] } \\
\hline \multicolumn{3}{|c|}{ Aggregation - Method II } \\
\hline \multirow{2}{*}{ Bus } & Filter Effect Neglected & Filter Modelled \\
\hline \multirow{2}{*}{$34.5 \mathrm{kV}$} & 18.03 & 5.62 \\
\hline $69 \mathrm{kV}$ & 9.84 & 3.29 \\
\hline
\end{tabular}

Despite of having a better result when comparing the impedance frequency response, the voltage distortion obtained from the first aggregation method did not meet with the expected.

The study case was also simulated focusing on the impact that the distribution system modelling has on the harmonic results. The wind farm was simulated with its internal system aggregated by the second method, which showed better results.

The first two cases simulated the wind farm with the transmission system seen from the PCC represented with the short-circuit impedance and the L-C-L circuit equivalent. The last two results were obtained based on a more detailed system modelling, where 19 buses from the EHV system was explicitly represented. This simulation 
permitted the analysis of the transmission line impedance effect, where it can be evaluated using the distributed parameter or the concentrated parameter model. The results obtained for these cases are summarized in Table VIII.

The harmonic current was recalculated according with (1) considering two different cases: $Z_{\text {grid }}$ obtained from a short-circuit power of $150 \mathrm{MVA}$ and $1500 \mathrm{MVA}$. The different current spectrums, illustrated in Figure 9, were simulated to compare the effect of this parameter on the result.

Table VII. - Transmission system modelled as an equivalent

\begin{tabular}{|c|c|c|c|}
\hline \multicolumn{4}{|c|}{ Short-circuit equivalent } \\
\hline \multirow{3}{*}{ Bus } & \multicolumn{3}{|c|}{ THD (\%) } \\
\hline & \multicolumn{2}{|c|}{ With capacitor } & \multirow{2}{*}{ Without capacitor } \\
\hline & Case I & Case II & \\
\hline $34.5 \mathrm{kV}$ & 9.31 & 8.48 & 55.89 \\
\hline $69 \mathrm{kV}$ & 4.32 & 4.17 & 34.21 \\
\hline $230 \mathrm{kV}$ & 0.84 & 0.85 & 8.61 \\
\hline \multicolumn{4}{|c|}{ L-C-L circuit equivalent } \\
\hline \multirow{3}{*}{ Bus } & \multicolumn{3}{|c|}{ THD $(\%)$} \\
\hline & \multicolumn{2}{|c|}{ With capacitor } & \multirow{2}{*}{ Without capacitor } \\
\hline & Case I & Case II & \\
\hline $34.5 \mathrm{kV}$ & 8.49 & 7.63 & 25.22 \\
\hline $69 \mathrm{kV}$ & 4.64 & 4.23 & 10.22 \\
\hline $230 \mathrm{kV}$ & 2.41 & 2.60 & 6.48 \\
\hline \multicolumn{4}{|c|}{ Transmission lines modelled with concentrated parameters } \\
\hline \multirow{3}{*}{ Bus } & \multicolumn{3}{|c|}{ THD $(\%)$} \\
\hline & \multicolumn{2}{|c|}{ With capacitor } & \multirow{2}{*}{ Without capacitor } \\
\hline & Case I & Case II & \\
\hline $34.5 \mathrm{kV}$ & 10.94 & 9.67 & 21.85 \\
\hline $69 \mathrm{kV}$ & 5.38 & 4.88 & 9.41 \\
\hline $230 \mathrm{kV}$ & 1.60 & 1.72 & 7.36 \\
\hline \multicolumn{4}{|c|}{ Transmission lines modelled with distributed parameters } \\
\hline \multirow{3}{*}{ Bus } & \multicolumn{3}{|c|}{ THD (\%) } \\
\hline & \multicolumn{2}{|c|}{ With capacitor } & \multirow{2}{*}{ Without capacitor } \\
\hline & Case I & Case II & \\
\hline $34.5 \mathrm{kV}$ & 9.26 & 8.15 & 27.77 \\
\hline $69 \mathrm{kV}$ & 4.75 & 4.34 & 20.01 \\
\hline $230 \mathrm{kV}$ & 1.82 & 1.89 & 11.08 \\
\hline
\end{tabular}

Considering the last case, with the transmission system represented with $19 \mathrm{EHV}$ busses and the transmission lines modelled with distributed parameters, as a more accurate result, it can be seen that assumptions made for the equipment modelling play an important role in harmonic penetration analysis.

Simulating the system without accounting with the WTG second-order low pass filter presented very severe results. Also, from the point of view of the grid, the difference in the filter gain by altering the short-circuit power, $Z_{\text {grid }}$ in (1), showed very little difference from each other. When comparing the first two results, the limitations of simpler representation are evident. Considering the THD at the 230 $\mathrm{kV}$ bus, when the system is modelled as a short-circuit impedance, the error presented is $53.4 \%$. With the L-C-L equivalent, the error for was as high as $37.6 \%$. The transmission line modelling affected very little the harmonic distortion results. The highest error observed for the THD at the $230 \mathrm{kV}$ bus was $12 \%$.

\section{Conclusions}

There are several ways to model an electrical system when analysing harmonic propagation. Although several studies propose a rule of thumb when dealing with electrical equipment representation, it always lacks a quantitative comparison against each possibility.

This paper simulates a wind power system with parameters estimated from commercial systems and analysed it, examining the overall impact of system modelling and the degree of representation.

For harmonic studies, cable and transformers models presented in power system analysis engineering software, e.g. DIgSILENT PowerFactory, are very accurate. The degree of representation of external system is important, representing it with the short-circuit impedance at the PCC is very poor. For the presented study case, the result converged for the expected result with the system modelled with 19 busses and considering only secondary distribution system connected to the PCC. Further studies must be carried out in order to verify the accuracy of the reduced representation in other parts of the Brazilian grid. The harmonic source modelling must be analysed beyond the current spectrum measured. Filters used to mitigate the harmonic propagation have an important effect on the system impedance, altering the study results. Another study using time-domain simulation could enhance the analysis of the filter impact.

\section{References}

[1] S.J. Ranade and W. Xu, "An Overview of Harmonic Modelling and Simulation", Tutorial Harmonics Modeling and Simulation, IEEE Power Engineering Society, 1998.

[2] J. Arrillaga and N.R. Watson, Power System Harmonics, John Willey and Sons, London (2003), pp. 261-348.

[3] CIGRE JTF 36.05.02/14.03.03, "AC System Modelling for AC Filter Design - An Overview of Impedance Modelling", ELECTRA No. 164, 1996.

[4] P.F. Ribeiro, "Guidelines on Distribution System and Load Representation for Harmonic Studies", in ICHPS V, pp. 272280, Atlanta, 1992.

[5] IEC 61400-21-2008, "Wind Turbine Generator SystemsPart 21: Measurement and Assessment of Power Quality Characteristics of Grid Connected Wind Turbines".

[6] B. Gustavsen, J. Martinez, and D. Durbak, "Parameter Determination for Modeling System Transients - part II: Insulated Cables", in IEEE Transactions on Power Delivery, vol. 20, no. 3, pp. 2045-2050, 2005.

[7] IEEE Std C37.011-2005, "IEEE Application Guide for Transient Recovery Voltage for AC High-Voltage Circuit Breakers Rated on a Symmetrical Current Bases", pp. 49-53.

[8] J. Li, N. Samaan, S. Willians, "Modeling of Large Wind Farms Systems for Dynamic and Harmonic Analysis", in Transmission and Distribution Conference and Exposition, pp. 1-7, 2008.

[9] F. Ghassemi and K. Kah-Leong, "Equivalent Network for Wind Farm Harmonic Assessments", in IEEE Transactions on Power Delivery, vol. 25, no. 3, pp. 1808-1815, 2010.

[10] E. Muljadi, et al, "Equivalencing the Collector System of a Large Wind Power Plant", in IEEE Power Engineering Society General Meeting, 2006. 